УДК 621.9.02

\title{
PRINCIPLE OF LOAD EQUALIZING IN SPLIT-POWER TRANSMISSION SYSTEM
}

\author{
(C) Stephen P. Radzevich \\ EATON Corp., Detroit, USA \\ radzevich@usa.com
}

\begin{abstract}
This paper deals with split-power transmission systems (or with SPTS, for simplicity). SPTS feature two or more paths of power flow. An increased power density is the fundamental advantage of SPTS over mechanical systems with a single power path. It is commonly assumed that in SPTS, the input power splits equally among all the power paths (or, more generally, it splits in specified proportions among the power paths). Because of the design features of SPTS, manufacturing errors, displacements under operating load, heat extension, etc., the input power is shared unequally among the power paths. Because of this, the components in some of the power paths are overloaded, while other ones are underloaded, or they can be even idle. As a result, efficiency of SPTS drastically goes down. Enhanced accuracy in the machining of the components is a straightforward, less-than-economical approach to equalizing the load share in SPTS. The problem of equal (or almost) equal load share in SPTS can be solved by means of elastic absorbers of manufacturing errors. Below in this paper, this approach is discussed in detail. In this research SPTS of two kinds are distinguished. They are symmetrical ( 2000), and asymmetrical SPTS ( 2011). In symmetrical SPTS equal load share is ensured by means of elastic absorbers of manufacturing errors (or means of EAME, for simplicity). In asymmetrical SPTS, the displacements of operating components as a result of asymmetry of SPTS. The unfavorable variation of the displacements can also be equalized by means of proper elasticity of the main components of SPTS. A few illustrative examples of application are presented.
\end{abstract}

Key words: SPTS (split-power transmission system), Equal load share, Power density, Power path.

\section{ПРИНЦИП ВЫРАВНИВАНИЯ НАГРУЗКИ В МНОГОПОТОЧНЫХ МЕХАНИЧЕСКИХ ПЕРЕДАЧАХ}

(C) Степан Павлович Радзевич

EATON Corp., Detroit, USA

radzevich@usa.com

\begin{abstract}
Аннотация. В статье рассматриваются многопоточные механические передачи (или, для простоты, МПМП). МПМП имеют два и более путей (потоков) передаваемой момности. Повышенная удельная мощность является основным преимуществом МПМП по сравнению с механическими системами с одним потоком передаваемой мощности. Обычно предполагается, что входная мощзность в МПМП распределяется равномерно между всеми потоками мощности (или, в более общем случае, она распределяется в предопределенных пропорииях между потоками мощности). Из-за конструктивных особенностей, производственных отклонений, смещений при рабочей нагрузке, теплового расширения $u$ т.д., входная мощность распределяется между потоками мощности неравномерно. Из-за этого компоненты в некоторых потоках мощности оказываются перегруженными, тогда
\end{abstract}


как другие недогружены или даже могут быть не вовлечены в передачу мощуности. В результате эффективность МПМП резко падает. Повышенная точность обработки компонентов - это простой, менее чем экономичный подход к выравниванию доли нагрузки в МПМП. Проблема выравнивания (или почти выравнивания) нагрузки в МПМП может быть решена с помощью упругих поглотителей производственных ошибок. Ниже в этой статье этот подход подробно обсуждается. В этом исследовании различают два вида МПМП. МПМП бывают симметричными ( 2000) и асимметричными ( 2011). B симметричных МПМП равномерное распределение нагрузки обеспечивается за счет эластичных поглотителей производственных погрешностей (или ЭППП, для простоты). $B$ несимметричных смещение рабочих компонентов имеет место в результате несимметричности МПМП. Неблагоприятное изменение перемещений можно также компенсировать за счет надлежащей эластичности основных компонентов. Приведено несколько наглядных примеров применения как симметричных МПМП, так $u$ несимметричных МПМП.

Ключевые слова: МПМП (многопоточная механическая передача), одинаковое распределение нагрузки, плотность мощчности, поток мощности.

Introduction. This paper deals with split-power-transmission-systems (or with SPTS, for simplicity). Transmission systems of this kind feature two or more paths of transmitted power. An increased power density is the fundamental advantage of SPTS over mechanical systems with single power path.

For decades the problem of equal power share among several power flows in SPTS is under investigation by mechanical engineering. Lots of efforts have been undertaken in the past to solve this problem. However, many efforts do not mean many results. The number of scientific publications on the topic is limited. Planetary reducers, automobile differentials and similar gear trains with split power are likely the most investigated examples of SPTS .

The earlier proposed by Prof. L.N. Reshetov, L.N. approach [5] is focused on application of the socalled self-aligning mechanisms for the purpose of equal load share in split-power-transmissionsystems. In order to neutralize an excessive mobility of some components, additional mobility of other components of SPTS are added following the approach [5]. In this way ultimate mobility of the SPTS equals to one. This makes SPTS more complex.

Use of elastic absorbers of manufacturing errors is the key point in the alternative approach of load equalizing in SPTS proposed by the Prof. S.P. Radzevich [1]. This approach is focused on elimination of excessive mobility of components in SPTS .

Latest author's accomplishments in this field of mechanical engineering are briefly outlined in the text immediately below. The discussion is focused on asymmetrical (Prof. S.P. Radzevich, 2011) and on symmetrical (Prof. S.P. Radzevich, 2000) split-power-transmission-systems.

1. Asymmetrical Split-Power-Transmission Systems. A large variety of kinds of split-powertransmission-systems is used in the nowadays industry. All SPTS fall into two groups. Asymmetrical SPTS comprise the first group, and symmetrical SPTS comprise the second group of kinds of split-power-transmission-systems. The second group of SPTS can be viewed as a reduced case of the first one.

1.1. Principal kinds and structural features of asymmetrical SPTS. In "asymmetrical splitpower-transmission-systems" (Prof. S.P. Radzevich, 2011) the actual values of the principal design parameters of SPTS are different in different power flows. Shown in Fig. 1 split-powertransmission-system, that is used in the design of automobile seat track, can be viewed as a representative example of asymmetrical SPTS. Because of the evident reasons, the electric motor cannot be located at the center of the seat cushion. Therefore, it is mounted at a side of the seat 12 
cushion. When the electric motor is on, the electric motor spindle rotates a long flex-shaft, and a short flex-shaft. Through two gearboxes, this rotation is transmitted to two screw-and-nut kinematic pairs that move the seat back and forth. The flexible shafts, namely the long one and the short one, are of different lengths (and this is inevitable). Actual lengths of the flex-shafts are the principal design parameters of the SPTS. Because the shafts are of different length, split-powertransmission-system of this particular design (see Fig. 1) is referred to as "asymmetrical SPTS".

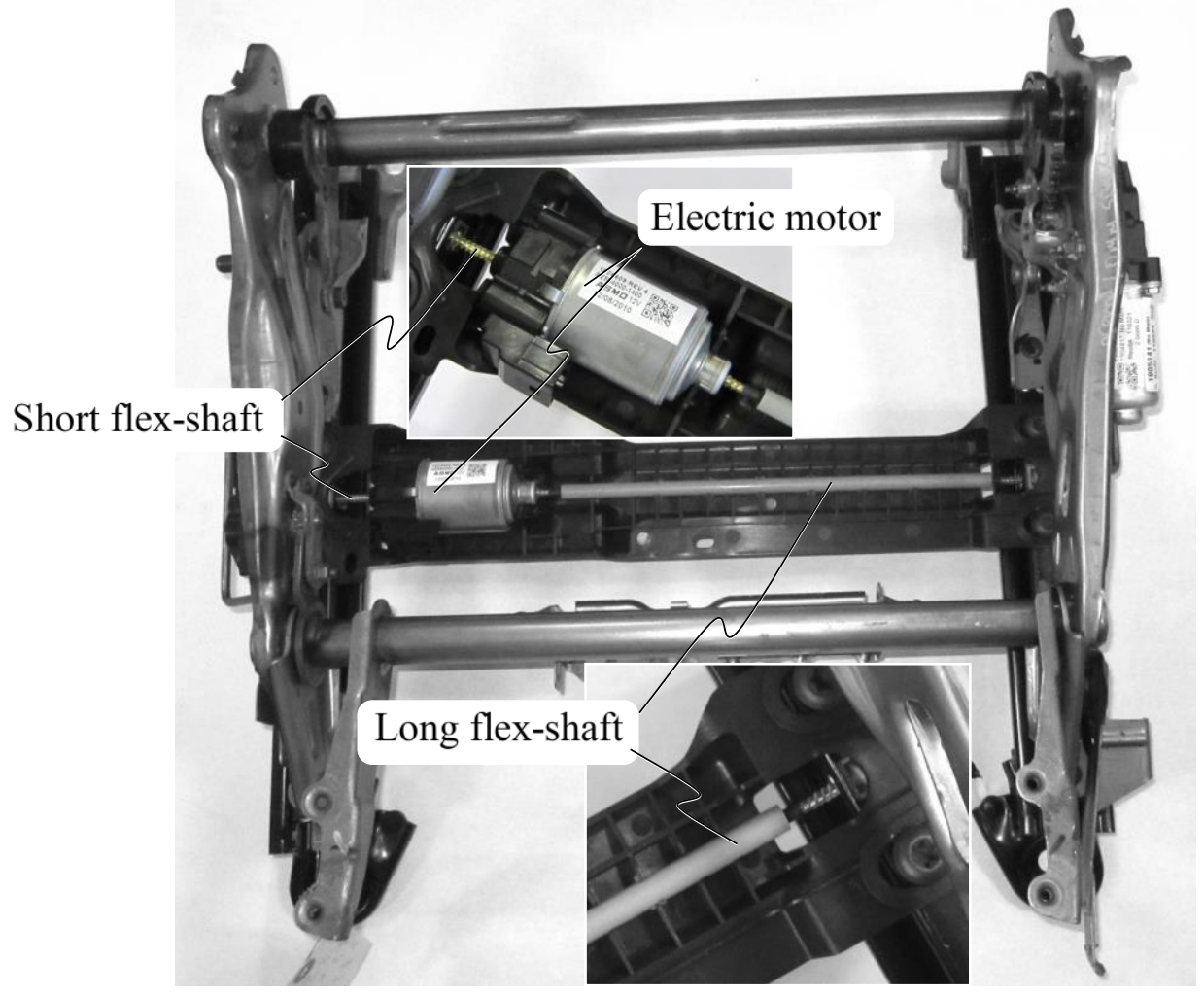

Fig. 1. Close-up of automobile seat track (representative example of asymmetric SPTS.

When a torque from the electric motor is applied, the flexible shafts are twisted at different twist angles. This is because the flex-shafts are of different lengths. As a result, mis-phasing in the screw-to-nut kinematic pairs causes unfavorable misalignment of the direction of the force that acts against the seat, and between the seat track rails. Ultimately, the traverse of the seat track gets skewed, the seat motion back and forth becomes unsmooth, and produces excessive noise. None of these is permissible. This the first and the main source of inconsistences of operation of the asymmetrical SPTS. Manufacturing errors, displacements of the components under operating load, heat extension of the components and so forth comprise the second source of inconsistences of operation of the asymmetrical SPTS (to be considered below in relation to symmetrical SPTS ). The main features of asymmetrical SPTS are, first of all, due to:

(a) either power being transmitting through every power path, $T R A N S_{i}$, is not of the same value;

(b) or one (or more) of the principal design parameters $L_{e m / t}$ and/or $L_{t / o e} \operatorname{differ(s)}$ from others;

(c) or both items (a) and (b) occur at the same time.

Asymmetrical SPTS of the kind (a) are out the scope of the paper. The readers' attention here is focused just on the analysis of asymmetrical SPTS that feature different principal design parameters $L_{e m / t}$ and/or $L_{t / o e}$.

Possible structures of asymmetrical SPTS under consideration are illustrated in Fig. 2. 
First, an asymmetrical SPTS may feature single source of input power [either an engine/motor, $E / M$, or other] and certain number, $n$, of operating elements $(O E)$ as it is schematically shown in Fig. 2,a. In this particular case, each operating element, $O E_{i}$, is powered by that same source of power, $E / M$. For the purpose of transmitting of power from $E / M$ to each $O E_{i}$, a corresponding transmission, $T R A N S_{i}$, is used. The total number of the transmissions, TRANS $S_{i}$, is equal to the total number of the operating elements, $O E$.

The input power from a single source of power is split onto numerous $(n)$ paths. asymmetrical SPTS of this kind feature equal power being transmitting through each power path.

A transmission that splits the input power between the main rotor, and the tail rotor of a helicopter is a good example of asymmetrical SPTS of this particular kind.

Second, an asymmetrical SPTS may feature multiple sources of the input power ( $n$ engines/motors in total) and a single operating element, $O E$. Structure of asymmetrical SPTS of this kind is illustrated in Fig. 2,b. In this second case, the operating element, $O E$, is powered by each engine/motor, $E / M_{i}$ simultaneously. For the purpose of transmitting of power from each $E / M_{i}$ to $O E$, a corresponding transmission, $T R A N S_{i}$, is employed. Total number of the transmissions is equal to the total number of the sources of input power, $E / M_{i}$. Through numerous $(n)$ power paths, input power from all the $E / M_{i}$ is combined into a single path of output power. Asymmetrical SPTS of this kind feature equal power being transmitting through each path of power.

In a particular case, asymmetrical SPTS may feature a single source of input power (single engine/motor, $E / M$ ), and a single operating element, $O E$, connected to each other by means of

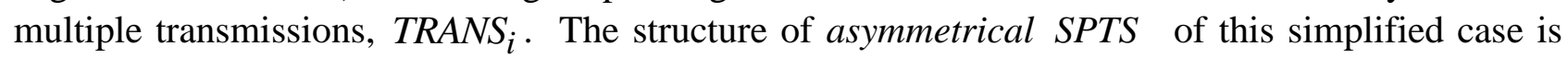
shown in Fig. 2,c. Again, asymmetrical SPTS of this kind feature equal power being transmitting

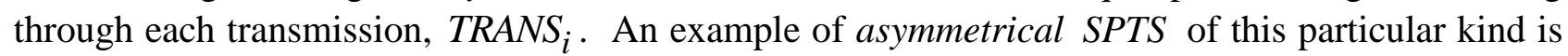
discussed immediately below.

1.2. Automobile seat track as an example of asymmetrical SPTS. Consider the design of an automobile seat track as an example of asymmetrical SPTS. An automobile seat track is a convenient example to illustrate possible approaches to ensure equal power share between two power flows in asymmetrical SPTS.

A close-up view of an automobile seat track is shown in Fig. 1. The seat track is composed of a motionless frame and of a horizontal motor bracket attached to the moveable slides. An electric motor is mounted at the top of the horizontal motor bracket. Both ends of the electric motor armature shaft are connected by two flexible shafts to two gear boxes (see Fig. 3). The transmission is attached to the slides. The flex-shafts are different in length. 

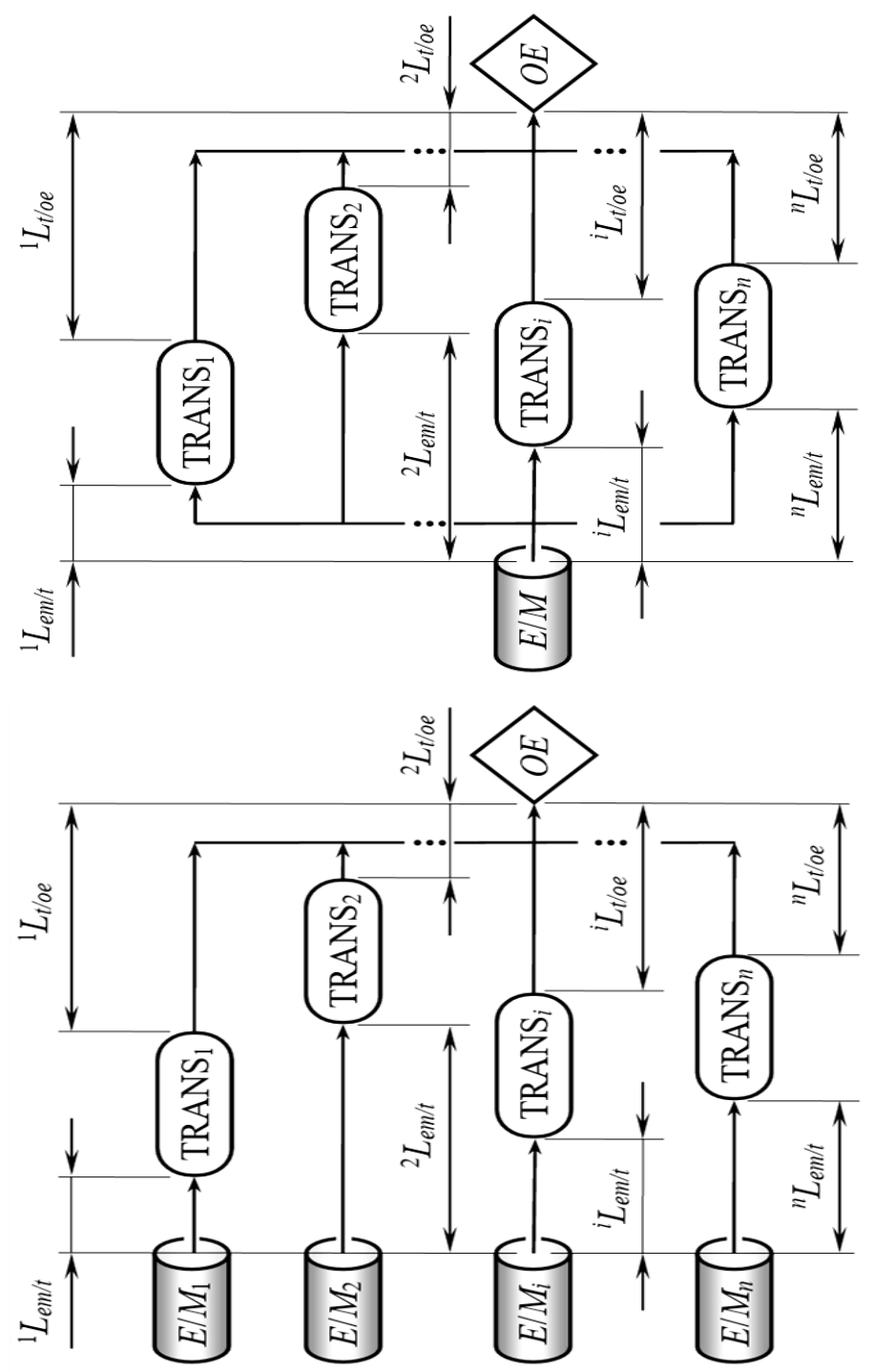

$\approx$

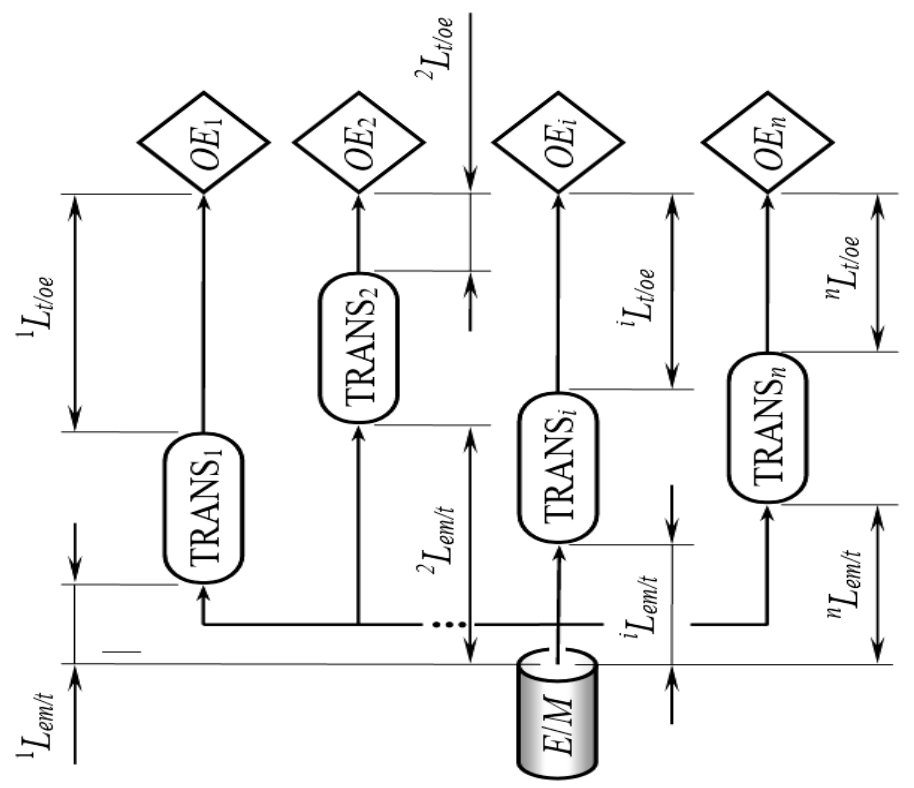

$\Xi$

Fig.2. Possible kinds of structure of asymmetric SPTS. 


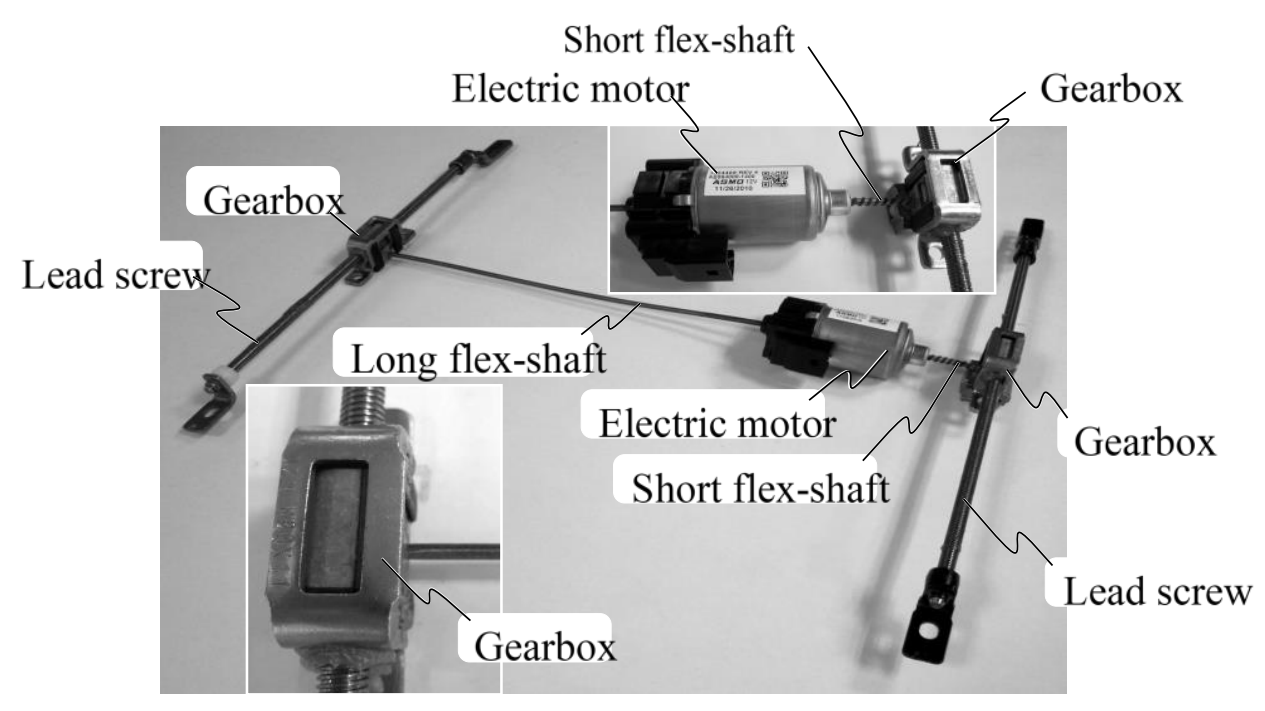

Fig. 3. Main components of asymmetric SPTS .

One of the flex-shafts (the long one) is supported by a sleeve, while another one (the short flexshaft) has no sleeve. The difference in length of the flex-shafts results in that the electric motor bracket got skewed immediately after the electric motor is turned on. In addition, stiffness of the flex-shafts also depends on direction of their rotation (either clockwise or counterclockwise), which is due to the actual direction of winding of wires when producing the flax-shafts.

In Fig. 3, the sleeve is removed from the long flex-shaft. Worm gear boxes are used in the design of the seat track. Tooth ratio of the worm gear box is equal to $u=7.5$. Two lead screws are riveted to the frame. External threads of the lead screws and internal threads in the worm of the worm gear box match to each other.

The occupant seat is rigidly connected to the horizontal motor bracket. When the horizontal motor bracket is moving, the occupant seat is moving back and forth as well.

When voltage is applied to the electric motor, power from the armature shaft is split between two ends of it. Rotation from the electric motor spindle is transmitted through the flexible shafts to two worm gear boxes. Then the reduced rotation of the worm goes to the lead screws. When the nut completes one full turn, then the horizontal motor bracket travels through the distance of $3 \mathrm{~mm}$. In this way the automobile seat is adjusted to a particular occupant.

The structure of asymmetrical SPTS of the seat track is schematically shown in Fig. 4.

It is desirable the power is split equally: half of the input power should go to the left-hand gear box, and then - to the worm and to the lead screw. Another half of the input power goes to the righthand gear box, and then - to the worm and to the lead screw. If this is ensured, the motor bracket is moving steady either straightforward, of backward.

1.3. Root causes of poor operating of automobile seat track. Several candidates for a potential root cause of poor operating of automobile seat track are recognized in the current design of the automobile seat track. Without going into details of consideration of the issue, the research below is focused just on one of them, namely, on difference between stiffness of the flexible shafts due to difference in length of them.

Under the operating load the flex-shaft is twisted through a twist angle, $\varphi$. The following formula:

$$
\varphi=\frac{T l}{J G}
$$

is commonly used to calculate the actual value of the twist angle, $\varphi$. 


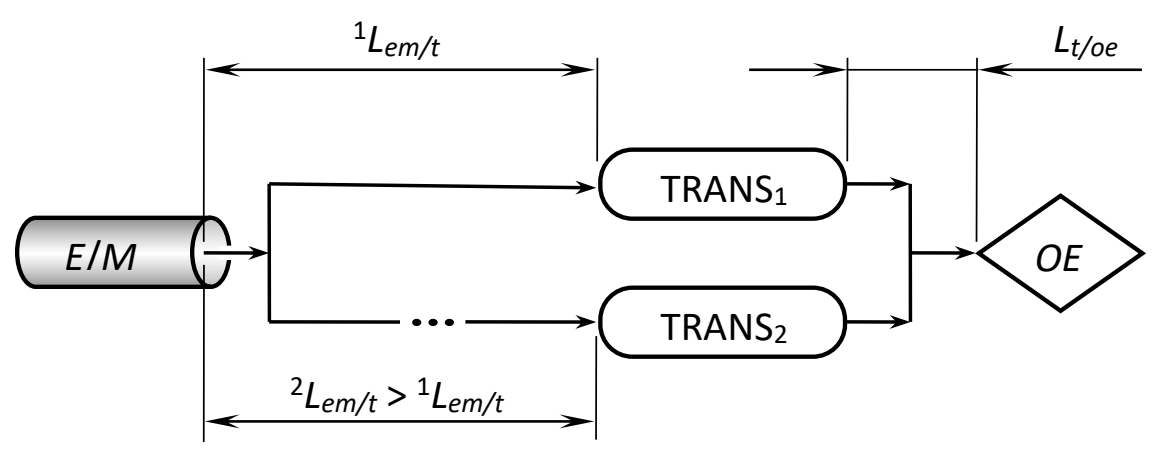

Fig. 4. Structure of asymmetric SPTS of an automobile seat track.

Here, in Eq. (1), is designated:

$T$ s the applied torque $\left(\mathrm{N}^{*} \mathrm{~m}\right)$

$l$ s the length of the flex-shaft

$J$ s the torsion constant for the section

$G$ s the shear modulus, or, more commonly, the modulus of rigidity and is usually given in gigapascals, GPa

The product $G J$ is commonly called the torsional rigidity.

As it follows from Eq. (1), the longer the flex-shaft, the larger the twist angle, $\varphi$, and vice versa.

It is right point to mention here that torsion constant for the section, $J$, is identical to the polar moment of inertia for a round shaft or concentric tube only. For other shapes $J$ must be determined by other means. For solid shafts the membrane analogy is useful, and for thin walled tubes of arbitrary shape the shear flow approximation is fairly good, if the section is not re-entrant. For most of the shapes there is no simple solution, and finite element analysis may be the best method.

When torque of a certain value $T_{i}$ is applied to the short flex-shaft, the flex-shaft is twisted through a corresponding twist angle, $\varphi_{i}^{s h}$. The twist angle, $\varphi_{i}^{s h}$, can be calculated from Eq. (1). The long shaft is connected to that same electric motor spindle. Therefore, when operating, the long shaft is twisted through the twist angle $\varphi_{i}^{l g}$. Evidently [see Eq. (1)], an inequality $\varphi_{i}^{l g}>>\varphi_{i}^{s h}$ is observed. As the twist angles, $\varphi_{i}^{s h}$ and $\varphi_{i}^{l g}$, the motor bracket.

1.4. Possible ways to improve the performance of asymmetrical STPS. In order to ensure high performance of the seat track it is necessary to ensure equal twist angles for both the flex-shafts. Several potential solutions to the problem under consideration a briefly outlined immediately below.

- The diameters of the long flex-shaft and of the short flex-shaft can be specified so as to keep an equality of the twist angles, $\varphi_{i}^{l g}=\varphi_{i}^{s h}$ : the longer flex-shaft, the larger its diameter and vice versa;

- The flex-shafts can be made up from different materials with different mechanical properties: the long flex-shaft is made up of more rigid material, while the short flex-shaft of more flexible material;

Numerous other approaches also allow for equalizing of the twist angles $\varphi_{i}^{s h}$ and $\varphi_{i}^{l g}$. However, at this point, the problem of unequal load share among all the power paths because of manufacturing errors of the components of symmetrical split-power-transmission-systems are composed, is remained unsolved. This issue is discussed in the section immediately below. 
2. Symmetrical Split-Power-Transmission Systems. Asymmetrical split-power transmission systems comprise another group of SPTS .

2.1. Principal kinds and structural features of symmetric SPTS. In "symmetrical split-powertransmission-systems" (Prof. S.P. Radzevich, 2000) the actual values of the principal design parameters of SPTS are identical in different power flows.

Symmetrical SPTS of two different kinds are distinguished in this paper.

First, a symmetrical SPTS may feature a single source of input power [either engine/motor ( $E$ / $M$ ), or other source of power], and certain number, $n$, of operating elements $(O E)$ as it is schematically shown in Fig. 5,a. In this particular case, each operating element, $O E_{i}$, is powered by that same source of power, $E / M$. For the purpose of transmitting of power from $E / M$ to every $O E_{i}$, a corresponding transmission, $T R A N S_{i}$, is employed. The total number of the transmissions is equal to the total number of the operating elements, $O E$.

The input power from a single source of power, $E / M$, is split onto numerous $(n)$ paths. Symmetrical SPTS of this kind feature equal power being transmitting through each path.

Examples of symmetrical SPTS of this particular kind can be found out in aerospace industry: gear transmission for the rotorcraft is a perfect example of symmetrical SPTS having the structure as that shown in Fig. 5,a.

Second, a symmetrical SPTS may feature multiple sources of input power [either engines/motors ( $n$ ) engines/motors, or other sources of power] and a single operating element, $O E$. The structure of symmetrical SPTS of this kind is depicted in Fig. 5,b. In this second case, the operating element, $O E$, is powered by several engine/motor, $E / M_{i}$ simultaneously. A corresponding transmission, TRANS $S_{i}$, is employed for the purpose of transmitting of power from each $E / M_{i}$ to $O E$. The total number of the transmissions, $T R A N S_{i}$, is equal to the total number of the operating elements, $O E$.

The input power from all the $E / M_{i}$ is combined into a common path of power.

Examples of complex mechanical systems of this particular kind can be found out in design of helicopter transmission where two jet engines rotate a single rotor.

In a particular case, symmetrical SPTS may feature a single engine/motor, $E / M$, and a single operating element, $O E$, connected to each other by means of multiple transmissions, TRANS . The structure of a symmetrical SPTS of this simplified case is shown in Fig. 5,c. A planetary gearbox is a good example of symmetrical SPTS of this particular kind.

It can be assumed that a hypothetical symmetrical SPTS can feature both, multiple sources of input power along with multiple operating elements. Besides no physical restrictions are imposed, symmetrical SPTS of this particular kind are out the scope of this paper.

In particular cases, symmetrical SPTS those illustrated in Fig. 5, may feature a single transmission. A possibility of such the simplification is illustrated in Fig. 6. Here, the structure of a symmetrical SPTS in Fig. 6, a corresponds to that shown in Fig. 5,a; the structure of a symmetrical SPTS in Fig. 6,b corresponds to that shown in Fig. 5,b; finally, the structure of a symmetrical SPTS in Fig. 6,c corresponds to that shown in Fig. 5,c. It is clear that a totally degenerate case in Fig. 6,c is trivial. 

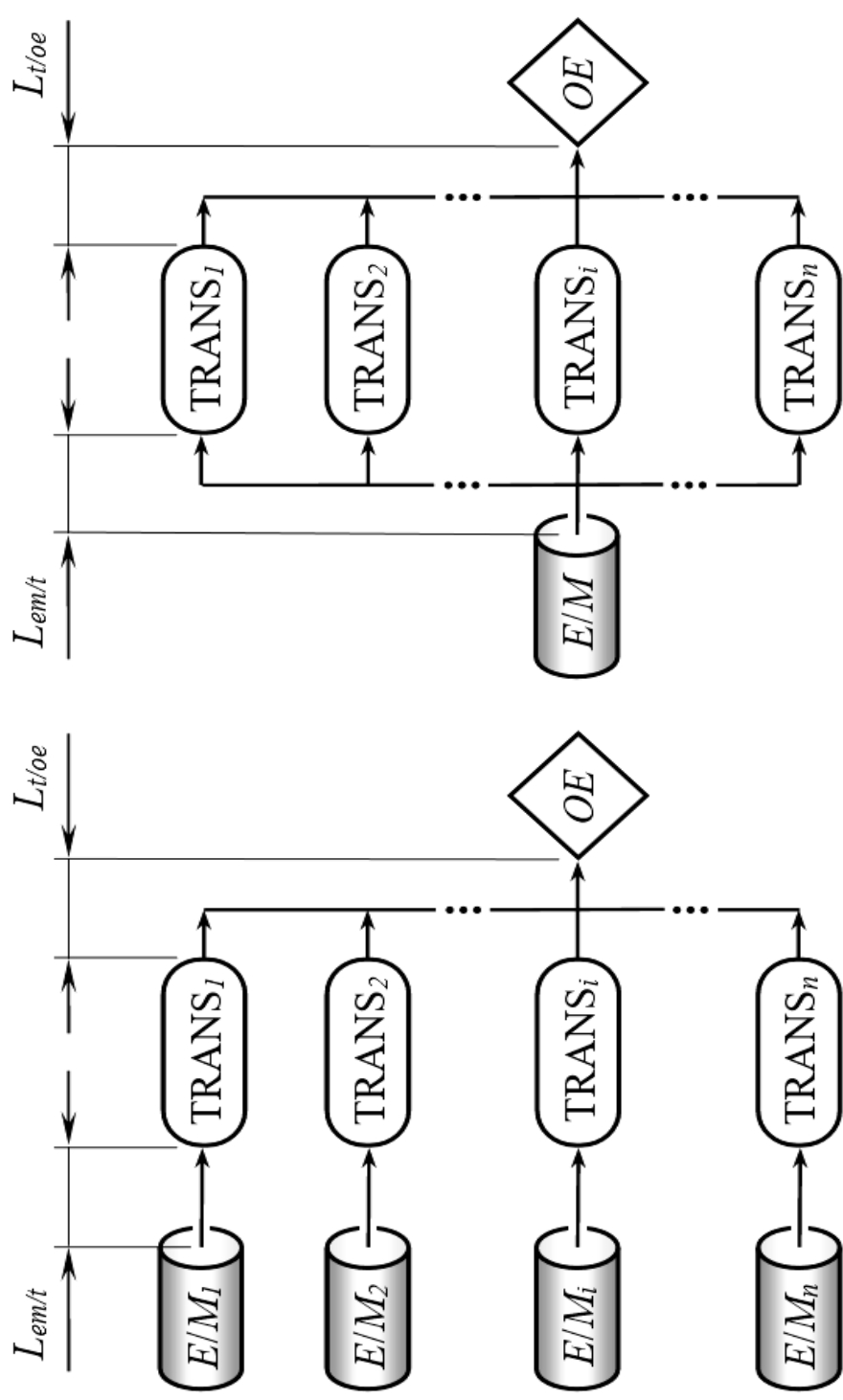

2

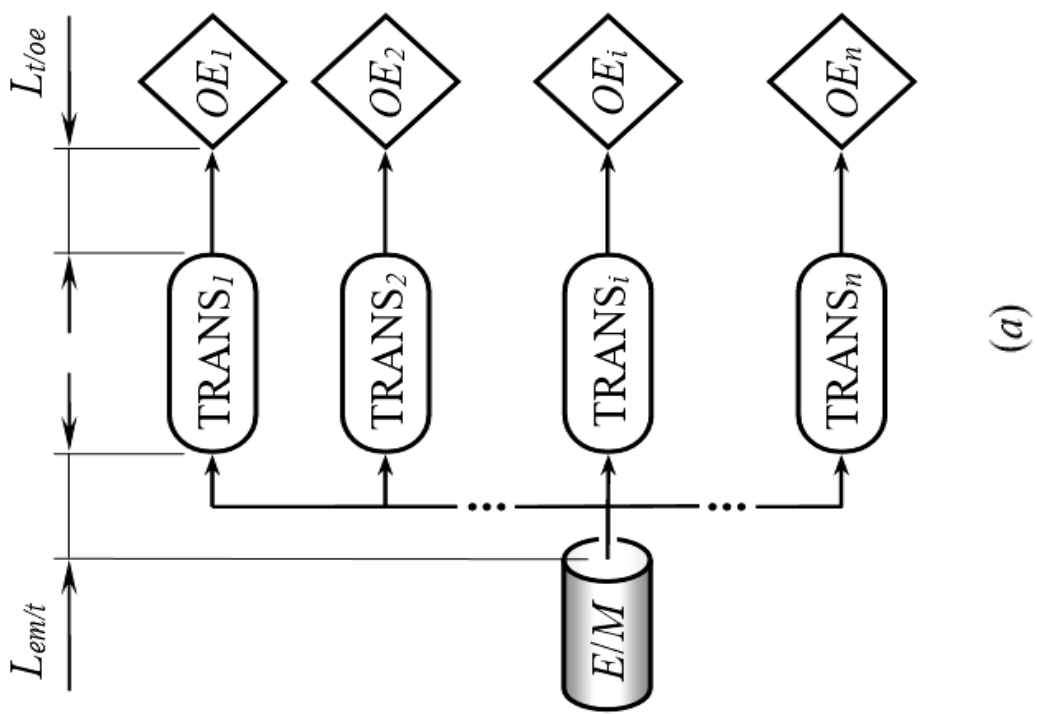

Fig.5. Possible kinds of structure of asymmetric SPTS. 
It is assumed in the above consideration that all the transmissions, TRANS $S_{i}$, are identical (or, at least, almost identical) to one another. In reality, they differ from one another only because of manufacturing tolerances for the accuracy of the components the transmissions are composed of.

It is also assumed here that mechanical, thermal and other properties of all the components of a symmetrical SPTS, those in-between the power source(s) and transmission(s), as well as inbetween a transmission and the operating element are identical to one another (however, in reality they differ from one another within the tolerance for the accuracy of corresponding dimension). The identical mechanical, thermal and other properties ensure identical behavior of each input and output shaft for every transmission, TRANS . $_{\text {. }}$

All the mechanical, thermal and other properties (those encountered in the analysis) of the components in-between the power sources and the transmissions are designated as $L_{e m / t}$. The same is valid with respect to the corresponding design parameter $L_{t / o}$ for the components of a symmetrical SPTS, those in-between the transmissions, TRANS $S_{i}$, and the operating element(s), $O E_{i}$.

The design parameters $L_{e m / t}$ and $L_{t / o e}$ are equal to all of the input and output shafts accordingly. Manufacturing errors are the root cause poor operation of symmetric SPTS.

2.2. Planetary gearbox as an example of symmetrical SPTS. Planetary gearboxes are extensively used in industry; examples are readily found in the automotive, aerospace, and other industries. (An example of a five-pinion planetary gearbox is shown in Fig. 7).

Planetary gearboxes with multiple planetary pinions enable a substantial reduction in the size and weight of the gear drive - concurrent with the number of pinions in the gearbox - and only when the transmitted load is equally shared among all the planet pinions.

Epicyclic gear systems have typically been equipped with straddle-mounted planetary pinions with pins supported on the input and output sides of the carrier. The torsional wind-up of the carrier, position accuracy of the pins, machining tolerances of the planetary gear system components and bearings clearances - all can contribute to poor load sharing among the planetary pinions, as well as misaligned gear contacts in the deflected state. In the traditional epicyclical gearing system, the distance between planetary pinion centerlines is specified by the design to be within a fixed range it is widely recognized that load sharing is not evenly distributed among planetary gear meshes. Further - stress, too, is distributed invariably at mesh points. Load sharing and stress distribution at each mesh point are heavily influenced by global design configuration; backlash tolerance; component design tolerances; manufacturing accuracy; component deflection; and thermal distortion. Figure 8 shows in exaggerated form that contact is made at the mesh point $K_{s g . p}$ of the planet pinion before any contact is made at the mesh points of the other planets (assuming ring gear makes contact with all pinions at points $\left.K_{s g . p}\right)$. In a rigid system, this condition imposes unbalanced loading among the planetary pinions.

2.3. Elastic absorber of manufacturing errors. Equal (or almost equal) load share among the planet pinion can be attained by means of high accuracy of all the components that comprise a planetary gearbox. This way is costly. Another possible way is to absorb the manufacturing errors of reasonable values by means of the so-called "elastic absorber of manufacturing errors" (or just " EAME", for simplicity). 


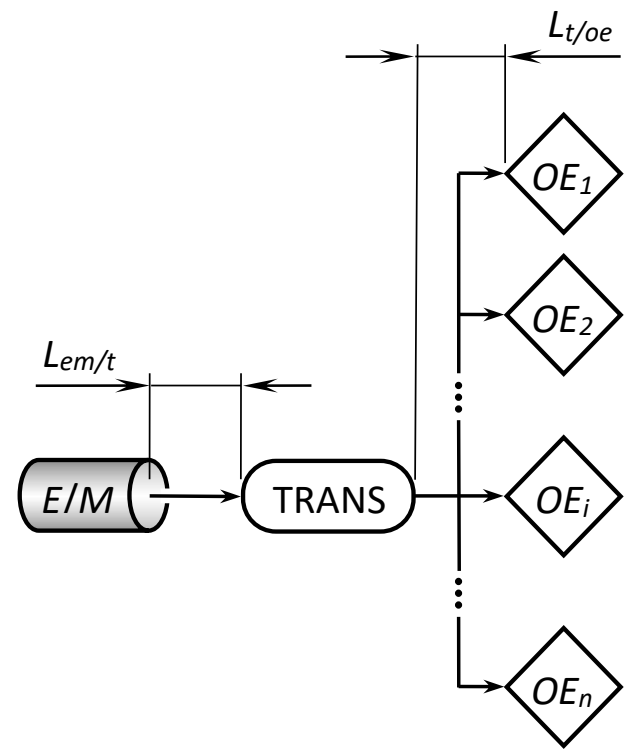

(a)

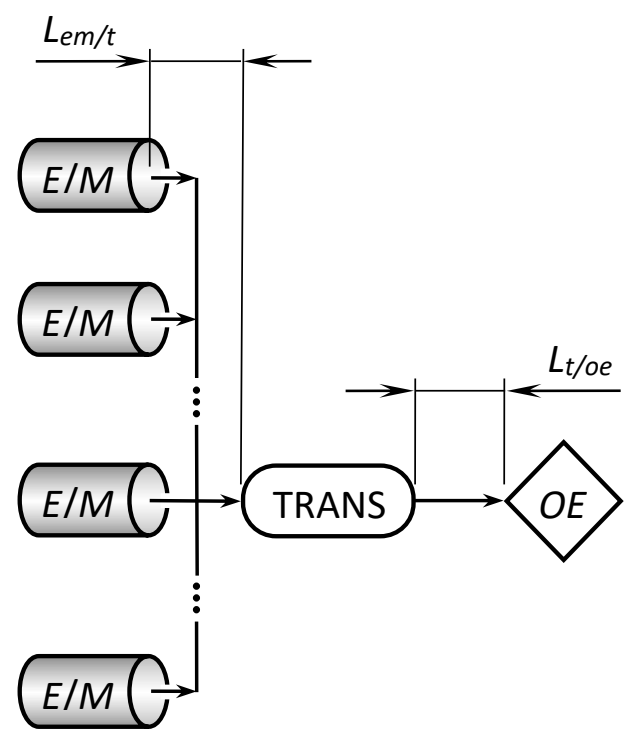

(b)

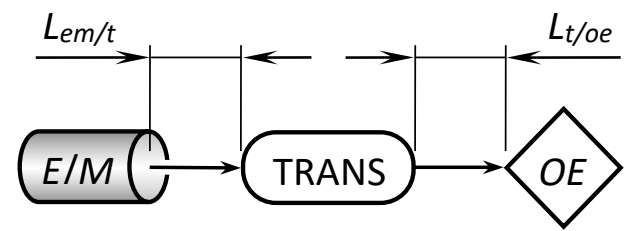

(c)

Fig. 6. Examples of simplified structures of complex symmetric mechanical systems. 


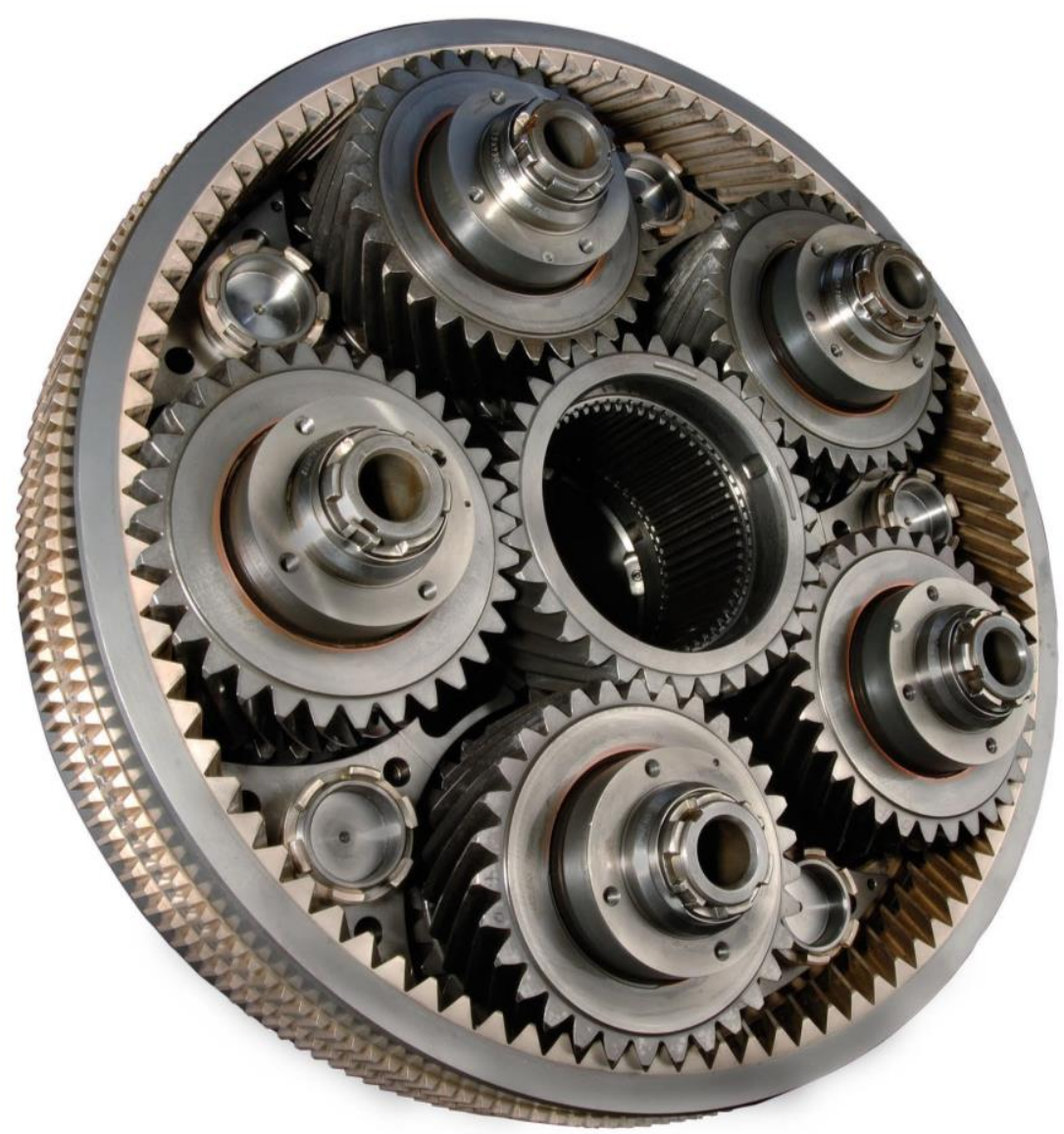

Fig. 7. Five-pinion planetary gearbox.

The purpose of the elastic absorbers of manufacturing errors is twofold:

1. to reduce the required minimum displacement of the planetary pinions

2. to make SPTS insensitive to manufacturing errors as well as to the displacements of other nature Therefore, the following features are inherited to $E A M E$ :

- $\quad$ EAME is elastic

- $\quad E A M E$ is pre-loaded by a pre-calculated load

- $\quad$ EAME is Mounted between any two components of the gear train where an additional degree-of-freedom is required to override the manufacturing errors.

If $E A M E$ is loaded by a pre-calculated value, the actual displacement of the pinions in the planetary gearbox (in the SPTS ) does not exceed the tolerance for the displacement.

Consider a planetary gearbox for which the permissible range of variation of load share among the planetary pinions is equal to $\pm O R_{l}$ (see Fig. 9, upper-left). The operating range of the deformation

$O R_{d}$ of the EAME needs to be the smallest possible; however, it must also be large enough to absorb the manufacturing errors, as well as the planetary pinions displacement under operating load. The two ranges $- \pm O R_{l}$ and $\pm O R_{d}$ - specify a rectangle. In Fig. 9, a diagonal of this rectangle forms an angle $\psi$ with the horizontal axis. The desirable stiffness " $c$ " of the EAME must be equal to (or less then):

$$
c \leq \tan \psi
$$

Once the stiffness " $c$ " is determined, a straight line through the origin can then be constructed. This line forms that same angle $\psi$ with the horizontal axis. The desirable pre-deformation of the 
EAME (see Fig. 9) is specified by point of interception of the constructed straight line and the straight line of the nominal (operating) load, $N L_{o p}$. The pre-deformation, $P D_{e a}$, of the EAME is calculated from the equation:

$$
P D_{e a}=\left(N L_{o p}-O R_{l}\right) \cdot \cot \psi
$$

Calculation of the design parameters of the elastic absorber of manufacturing errors is based on two parameters, namely, on the $\pm O R_{l}$ and on the $\pm O R_{d}$.

2.4. Illustrative example of application of $\boldsymbol{E A M E}$. In a two-stage planetary gearbox, the preloaded elastic absorber of manufacturing errors can be mounted in between the first-stage planet pinion and the second-stage planet pinion ${ }^{1}$ (see Fig. 10). It is common practice to hob both the planet pinions of the cluster planet pinion. For this purpose, it is convenient to assemble the cluster planet pinion comprising two planet pinions. However, note that proper phasing of the pieces in relation to one another while assembling the cluster planet pinion is critical; mis-phasing errors with planet pinions can be catastrophic. The preloaded elastic absorber of the manufacturing errors is installed between the two planet pinions of the cluster planet pinion (see Fig. 10).

For equal torque share among the planetary pinions, the misphasing $\Delta \varphi$ must be zero. As the misphasing $\Delta \varphi$ cannot be eliminated, it must be absorbed. For this purpose, it is necessary to eliminate all the excessive degrees of freedom for one of the planetary pinions in relation to another. The planet pinions can be made self-adjustable by meams of implementation of the preloaded elastic absorber of manufacturing errors. An angular displacement, $\Delta \varphi$, to be absorbed by the $E A M E$, can be eliminated, when the linear displacement, $\Delta l$, is equal to:

$$
\Delta l=\Delta \varphi \cdot r \cdot \frac{\pi}{180^{\circ}}
$$

In Eq, (4), the radial location of the EAME is specified by the distance $r$. Deformation, $\Delta l$, of an elastic body under load, usually (but not necessarily) relates to the applied load, $T$, linearly or (at least) almost linearly, $\Delta=c \cdot T$ ( $c$ is a porportionality factor equal to the rigidity of the preloaded elastic absorber). In such a case, the angle $\phi$ can be calculated from the formula:

$$
\phi=\tan ^{-1}(c)
$$

In the general case, when $c \neq$ const, the current value of $c$ is equal to $c=\frac{d T}{d(\Delta l)}$.

The interval of bariation of the applied load should be known for the calculation of the design parameter of the $E A M E$.

It should be stressed here that: Elastic absorbers of manufacturing errors can be used in design of split-power-transmission-systems (including, but not limited to planetary gearboxes) of any and all kinds, and sizes: of small, medium, as well as of large size.

Numerous split-power-transmission-systems are designed based on the approach outlined in this paper [2], [3], [4], as well, as many others.

\footnotetext{
${ }^{1}$ Radzevich, S.P., Planetary Reducer, Invention disclosure, filed to New Venture Gear, Inc., Patent Office (Syracuse, NY) on October 30, 2001, patent pending.
} 


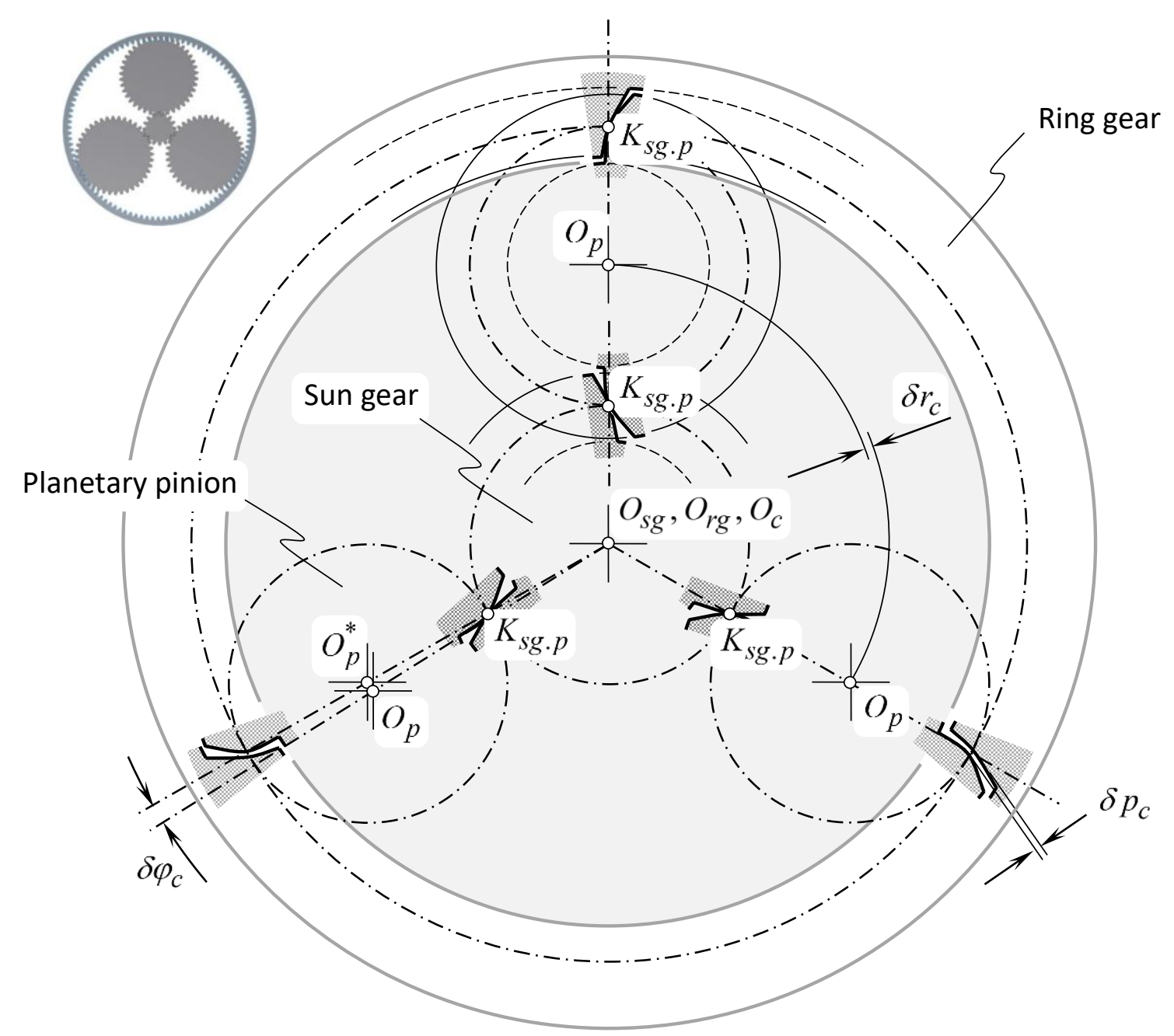

Fig. 8. Deviation of the actual position of planet pinion axis of rotation $O_{p}^{*}$ from its desirable position, $O_{p}$.

Conclusion: The paper deals with complex mechanical transmissions that feature plurality of paths of power flow. Split-power-transmission-systems, or just SPTS (for simplicity) is a common term for mechanical transmissions of this kind.

Two principal kinds of split-power-transmission-systems are distinguished. They are symmetrical and asymmetrical kinds of SPTS (Prof. S.P. Radzevich, 2000). Structural analysis of complex symmetrical as well as asymmetrical SPTS is performed. Significant similarities, as well as significant differences between SPTS of these two kinds. It is shown that synchronizing of the principal design parameters for all the power paths is the reliable way to improve the performance of asymmetrical SPTS (Prof. S.P. Radzevich, 2011).

A brief overview of known approaches for equal power sharing in planetary gearboxes is presented. A novel method for equal power sharing in planetary gearboxes is proposed. The method is based on use of the so-called "elastic absorbers of manufacturing errors", of just EAME, for simplicity. An illustrative example of the application of EAME is provided. Use of the concept of elastic absorbers of manufacturing errors allows a significant improvement in power density being transmitted through the planetary gearbox. The elastic absorbers of manufacturing errors can be applied in the design of multi-flow gear trains of any type. 


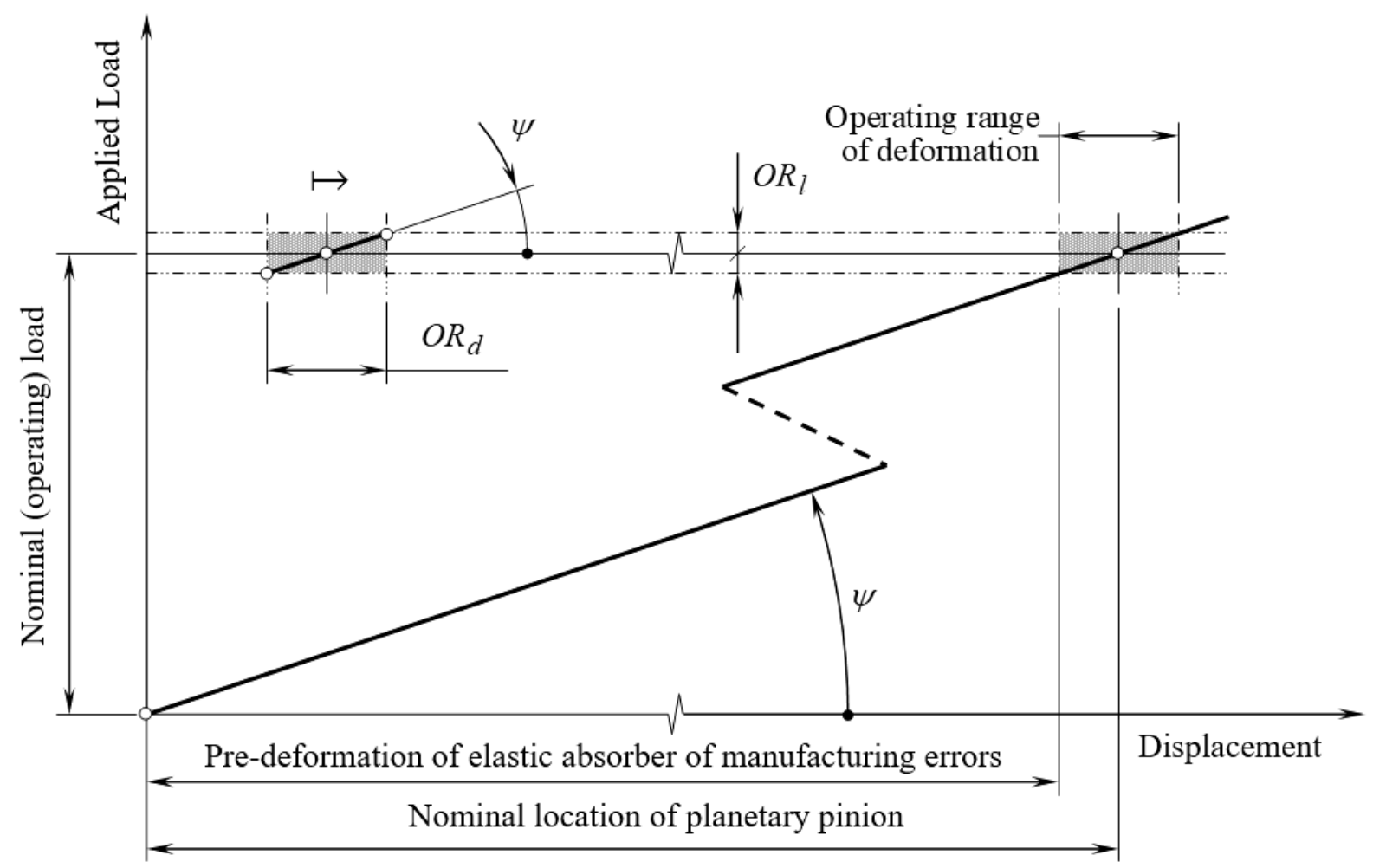

Fig. 9. Determination of principal design parameters of elastic absorber of manufacturing errors (After Prof. S.P. Radzevich, circa 2000, New Venture Gear, Syracuse, NY). 


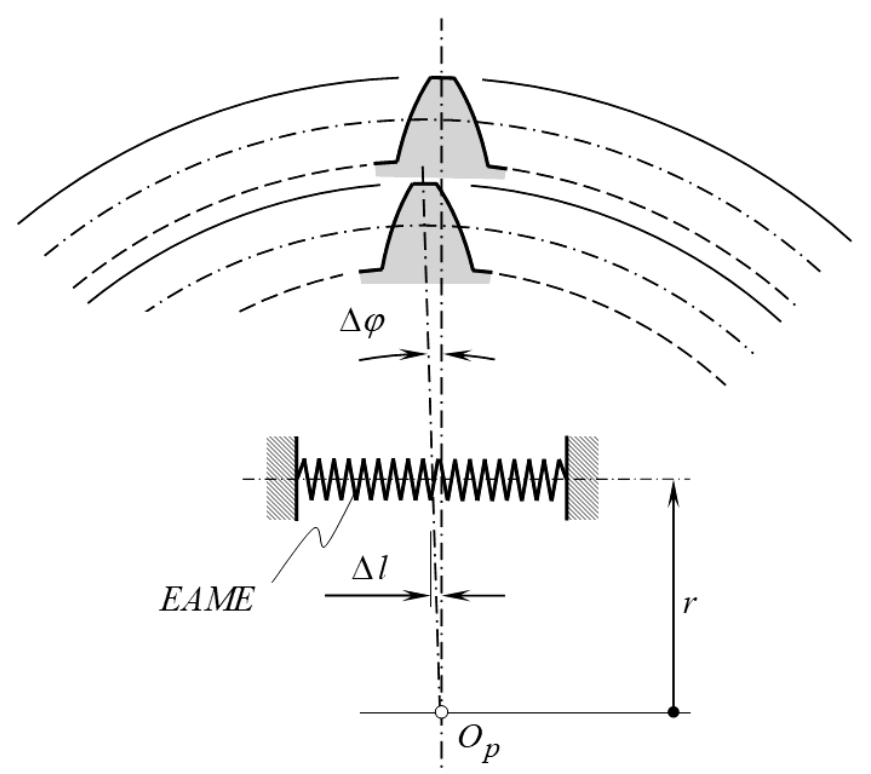

\section{Second-stage planet pinion}

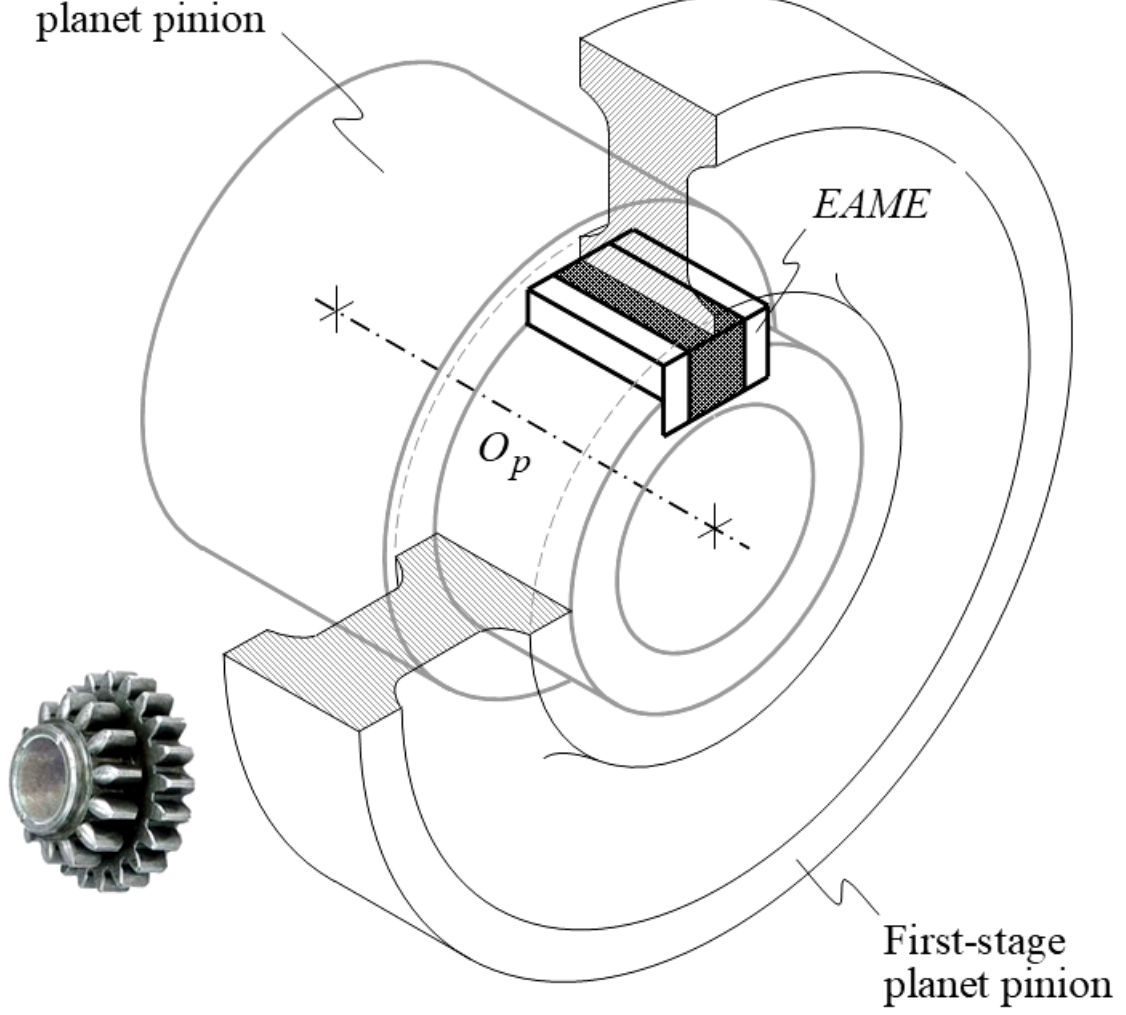

Fig. 10. Example of application of EAME in the design of cluster planet pinion (as proposed by Prof. S.P. Radzevich around 2000, New Venture Gear, Syracuse, NY). 


\section{References}

[1] Radzevich, S.P., Theory of Gearing: Kinematics, Geometry, and Synthesis, $2^{\text {nd }}$ Edition, revised and expanded, $C R C$ Press, Boca Raton, FL, 2018, 934 pages.

[2] Radzevich, S.P., et al, Gear Train with Split Torque, United States Patent Application Publication No. US 2010/0261568 A1, EATON Corp., Int. Cl. F16h 48/06, F16h 1/16, U.S. Cl. 475/226; 74/25 Filed: April 14, 2009, Published: October 14, 2010.

[3] Radzevich, S.P., et al, Face Gear Differentials Incorporating Torque Ring, United States Patent Application Publication No. US 2010/0317489 A1, EATON Corp., Int. Cl. F16h 48/06, F16h 55/17, U.S. Cl. 475/220; 74/458 Filed: June 14, 2010, Published: December 16, 2010.

[4] Radzevich, S.P., et al, Limited Slip Differential using Face Gears and a Pinion Housing, United States Patent Application Publication No. US 2010/0317482 A1, EATON Corp., Int. Cl. F16h 48/06, U.S. Cl. 475/226; Filed: April 15, 2010, Published: December 16, 2010.

[5] Reshetov, L.N., Self-Aligning Mechanisms, Translated from Russian by L.M. Sachs, Mir Publishers, Moscow, 1982, 582 p.

Дата поступления: 18 ноября 2020 г. 